\title{
Profile of patients admitted to a triage dermatology clinic at a tertiary hospital in São Paulo, Brazil ${ }^{*}$
}

\author{
Fernanda Bertanha ${ }^{1}$ \\ Alyne Korukian Freiberg ${ }^{1}$ \\ Cyro Festa Neto ${ }^{1}$
}

\author{
Erica Judite Pimentel Nelumba ${ }^{1}$ \\ Luciana Paula Samorano ${ }^{1}$
}

DOI: http:/ / dx.doi.org/10.1590/abd1806-4841.20164495

\begin{abstract}
BACKGROUND: Knowledge of epidemiological data on skin diseases is important in planning preventive strategies in healthcare services.

OвJестіvе: To assess data from patients admitted to a triage dermatology clinic.

MetHods: A retrospective study was performed of patients admitted over a one-year period to the Triage Dermatology Clinic at the Hospital das Clínicas of the University of São Paulo Medical School. Data were obtained from record books. The variables analyzed were: patient age, gender, dermatologic disease (initial diagnosis), origin (from where the patient was referred) and destination (where the patient was referred to).

REsults: A total of 16,399 patients and 17,454 diseases were identified for analysis. The most frequent skin disorders were eczema $(18 \%)$, cutaneous infections (13.1\%), erythematous squamous diseases $(6.8 \%)$ and malignant cutaneous neoplasms (6.1\%). Atopic dermatitis was the most common disease in children. Acne was more common among children and adults, as were viral warts. Basal cell carcinoma and squamous cell carcinoma were more common in the elderly. Contact dermatitis and acne predominated in women. The most frequent origins were: the primary/secondary health system (26.6\%), other outpatient specialties (25.5\%), emergency care $(14.9 \%)$; while the destinations were: discharged (27.5\%), follow-up in our Dermatology Division (24.1\%), return (14.1\%) and the primary/secondary health system $(20.7 \%)$.

CONCLUSION: Understanding the incidence of skin diseases is fundamental in making decisions regarding resource allocation for clinical care and research. Thus, we believe our findings can contribute to improving public health policies.
\end{abstract}

Keywords: Dermatology; Eczem; Epidemiology; Public health; Triage

\section{INTRODUCTION}

The skin is the outermost part of the human body and there is a huge variety of skin disorders. However, it is difficult to determine the exact prevalence or incidence of skin diseases due to a lack of epidemiologic studies on the general population. Many skin diseases are treatable but have a detrimental effect on quality of life. Knowledge of these epidemiological data is important in planning therapeutic and preventive strategies in healthcare services. ${ }^{1,2}$

This is highlighted to a greater or lesser degree in various studies. Penãte et al. conducted a study evaluating 3,144 applications for inpatient dermatology consultations at the Insular de Las Palmas de Gran Canaria University Hospital, Spain, concluding that the most frequent diagnoses were: contact dermatitis $(8.9 \%)$, drug reactions $(7.4 \%)$, candidiasis $(7.1 \%)$ and seborrheic dermatitis (5.3\%). ${ }^{3}$

At the Hospital das Clinicas of the University of São Paulo Medical School (HCFMUSP), 313 requests for referral to the dermatology division were evaluated; the most frequent diagnostic groups were infectious diseases, eczema and drug reactions. ${ }^{4}$

Inpatient treatment plays a fundamental role in managing complex and severe dermatological diseases. Rapid detection and diagnosis of findings can decrease morbidity, mortality, length of hospital stay and hospitalization costs. As with the data on inpatients, we analyzed the medical records of 3,308 patients

Received on 23.02.2015

Approved by the Advisory Board and accepted for publication on 05.07.2015

Work performed at the Departamento de Dermatologia do Hospital das Clínicas da Faculdade de Medicina da Universidade de São Paulo (HC-FMUSP) - São Paulo, Brazil.

Financial support: None.

Conflict of interest: None.

Universidade de São Paulo (USP) - São Paulo (SP), Brazil.

C2016 by Anais Brasileiros de Dermatologia 
hospitalized in the dermatology ward at the HCFMUSP for 8 years. Most admittances were for eczematous disease/dermatitis and skin infections. ${ }^{5}$ Bale $\mathrm{J}$ et al. conducted a survey at a tertiary referral hospital in New South Wales, Australia, examining 97 inpatient admissions in their dermatology unit during 2011. The most frequent reasons for admission were dermatitis or eczema and ulcers; the latter diagnosis accounted for the longest length of stay. ${ }^{6}$

Moreover, it is important to analyze skin disease patterns in the community outpatient setting to discuss strategies and the population's health demands. Some studies have been conducted in different countries based on this approach. In Japan, a nationwide multicentric survey was carried out, leading to the observation that the vast majority of the 67,448 cases evaluated involved miscellaneous eczema, atopic dermatitis and tinea pedis. ${ }^{1}$

Brazil has a decentralized public health system divided into three levels of care (primary, secondary and tertiary), in which patients are referenced according to the severity and complexity of the diagnosis. ${ }^{7}$ With this in mind, the General and Didactic Dermatology Clinic (AGDD) - a triage service - was developed at the HCFMUSP. This clinic is used to screen patients at the three different levels. Dermatology residents, medical students and residents of other specialties, supervised by assistant professors and chief residents, provide patient care. This study sought to assess epidemiologic data for patients admitted to the AGDD of the Dermatology Division at the HCFMUSP. The survey was designed as a relevant source of information for evaluating the frequency of dermatologic diseases at a public health service in Brazil.

\section{METHODS}

We performed a retrospective study of patients admitted to the AGDD of the HCFMUSP during a period of one year, from January $21^{\text {st }}, 2011$ to January $21^{\text {st }}$, 2012.

As a triage clinic, the AGDD experiences significant daily demand. Patients are evaluated and data are gathered in record books. Each patient can have up to three appointments, but most of them can be treated at the first visit. Patients can be referred from: the HCFMUSP's emergency unit, other outpatient specialties at the HCFMUSP or the primary and secondary health systems. Following the consultation, patients can be sent to the primary health system or the secondary health system. Alternatively, they can return for another evaluation at AGDD, follow-up in a specific group at the HCFMUSP's Dermatology Division or be discharged.

Data were obtained from record books. The variables analyzed were: patient age, gender, derma- tologic disease (initial diagnosis), origin (from where the patient was referred) and destination (where the patient was referred to after the appointment). Initial diagnoses were performed based on anamnesis and clinical signs. Direct microscopies of skin scrapings were carried out for relevant patients. We excluded patients who were on their return visit (repeated subjects) and those whose information regarding gender, age and diagnosis was unclear and/or missing.

Continuous variables were expressed as means with the respective standard deviation (SD). The descriptive analysis of categorical variables was calculated as absolute (n) and relative (\%) frequencies. We used two softwares, Microsoft Excel and SPSS 20.0 for MAC, to perform the analyses.

The HCFMUSP's Ethics Committee approved this study.

\section{RESULTS}

\section{Demographics}

During this one-year period under study, a total of 19,445 appointments were made at the AGDD, with 18,016 patients evaluated. Meanwhile, 302 subjects were excluded due to incomplete data and 1,315 were excluded because of indefinite diagnoses, meaning that the diagnostic hypotheses for the same skin lesion were from different categories of diagnosis, or that patients presented with uncharacteristic skin lesions which hampered the categorization of diagnosis. The remaining 16,399 subjects and their 17,454 diseases were also analyzed. There were 10,364 (63.2\%) females and $6,035(36.8 \%)$ males. The average age of subjects was 43.9 years (42.7 for males and 44.5 for females) with a SD of 22.1 (Table 1). The most frequent origins of patients were: the primary/secondary health system $(26.6 \%)$, other outpatient specialties $(25.5 \%)$ and Emergency Care (14.9\%). Their most common destinations were: discharged (27.5\%), follow-up in Dermatology Division of HCFMUSP (24.1\%), return (14.1\%) and the primary/secondary health system (20.7\%).

TABLE 1: Characteristics of patients

\begin{tabular}{ll}
\hline Subjects, $\mathrm{n}$ & 16,399 \\
Diseases, $\mathrm{n}$ & 17,454 \\
Patient age, years, mean \pm SD & $43.9 \pm 22.1$ \\
Gender considering subjects, $\mathbf{n}(\%)$ & \\
Female & $10,364(63.2 \%)$ \\
Male & $6,035(36.8 \%)$
\end{tabular}

$\mathrm{SD}=$ standard deviation 


\section{Dermatologic diagnosis}

All appointments were assigned to one or two of the following 15 categories of diagnosis: eczema, cutaneous skin infections, erythematous squamous diseases, malignant cutaneous neoplasms, benign cutaneous neoplasms, dyschromias, pruritic papular eruptions, nevi, trichosis, folliculosis, connective tissue disorders, nail disorders, asteatosis, other skin diseases and without skin disease. Eczema was the most frequent $(18.1 \%)$, and in most cases Non-specified $(7.4 \%)$, followed by: cutaneous infections (13.1\%; mostly superficial mycosis - $6.8 \%$ ), erythematous squamous diseases $(6.8 \%$, primarily psoriasis $-3.5 \%)$, miscellaneous malignant skin tumors $(6.1 \%$, including basal cell carcinoma (BCC) - 3.8\%) and miscellaneous benign skin tumors $(6.1 \%$, predominantly seborrheic keratosis $-3.6 \%$ ) (Table 2).

TABLe 2: Prevalence of skin diseases $(n=17,454)$.

\begin{tabular}{|c|c|c|c|c|c|}
\hline \multirow{2}{*}{$\begin{array}{l}\text { Frequency (n) } \\
\text { Eczema }\end{array}$} & \multicolumn{2}{|c|}{ Frequency \% } & \multirow{2}{*}{$\begin{array}{l}\text { Frequency }(\mathrm{n}) \\
\text { Pruritic papular eruption }\end{array}$} & \multicolumn{2}{|c|}{ Frequency $\%$} \\
\hline & 3,153 & 18.1 & & 620 & 3.5 \\
\hline Non-specified eczema & 1,301 & 41.3 & Insect bite reactions & 232 & 37.5 \\
\hline Atopic dermatitis & 593 & 18.8 & Graft-Versus-Host Disease (GVHD) & 122 & 19.7 \\
\hline Contact dermatitis & 476 & 15.1 & Lichen Striatus & 115 & 18.6 \\
\hline Lichen simplex chronicus & 315 & 10.0 & Lichen nitidus & 114 & 18.4 \\
\hline Others & 468 & 14.8 & Others & 35 & 5.7 \\
\hline Cutaneous infections & 2,293 & 13.1 & Nevi & 590 & 3.4 \\
\hline Superficial mycosis & 1,182 & 51.5 & Melanocytic nevi & 222 & 37.7 \\
\hline Viral warts & 372 & 16.2 & Melanosis Solar & 145 & 24.5 \\
\hline Abscess, furuncle and anthrax & 120 & 5.2 & Non-specified nevi & 138 & 23.5 \\
\hline Molluscum contagiosum & 112 & 4.9 & Ephelis & 13 & 2.2 \\
\hline Others & 507 & 22.1 & Others & 72 & 12.2 \\
\hline Erythematous Squamous Dermatosis & 1.197 & 6.9 & Trychosis & 587 & 3.4 \\
\hline Psoriasis & 646 & 54.0 & Alopecias areata & 173 & 29.4 \\
\hline Seborrheic dermatitis & 363 & 30.3 & Androgenic alopecia & 103 & 17.6 \\
\hline Pityriasis rosea & 136 & 11.4 & Telogen effluvium & 90 & 15.3 \\
\hline Non-specified erythematous squamous dermatosis & 24 & 2.0 & Non-specified trychosis & 82 & 13.9 \\
\hline Others & 27 & 2.3 & Others & 139 & 23.8 \\
\hline Malignant cutaneous neoplasms & 1,071 & 6.1 & Foliculosis & 556 & 3.2 \\
\hline Basal cell carcinoma & 665 & 62.1 & Acne & 433 & 77.8 \\
\hline Squamous cell carcinoma & 203 & 19.0 & Rosacea & 103 & 18.5 \\
\hline Melanoma & 106 & 9.9 & Dermatitis perioralis & 20 & 3.6 \\
\hline Non-specified malignant cutaneous neoplasms & 96 & 9.0 & Connective Tissue Disorders (Fat, Muscular or Neural) & 530 & 3.0 \\
\hline Others & 1 & 0.1 & Soft fibroma/acrochordon & 157 & 29.6 \\
\hline Benign cutaneous neoplasms & 1,060 & 6.1 & Keloid & 152 & 28.6 \\
\hline Seborrheic keratosis & 628 & 59.2 & Lipoma & 83 & 15.6 \\
\hline Actinic keratosis & 291 & 27.5 & Dermatofibroma & 56 & 10.5 \\
\hline Dermatosis papulosa nigra & 38 & 3.6 & Others & 83 & 15.6 \\
\hline Non-specified benign cutaneous neoplasms & 38 & 3.6 & Nail Disorders & 417 & 2.4 \\
\hline Others & 65 & 6.1 & Non-specified nail disorders & 158 & 37.8 \\
\hline Dyschromias & 748 & 4.3 & Onicocriptosis & 103 & 24.7 \\
\hline Vitiligo vulgaris & 298 & 39.9 & Chronic Paronychia & 89 & 21.3 \\
\hline Melasma & 212 & 28.4 & Onychodystrophy & 46 & 11.1 \\
\hline Pityriasis alba & 98 & 13.1 & Others & 21 & 5.1 \\
\hline Non-specified dyschromias & 82 & 10.9 & Asteatosis & 365 & 2.1 \\
\hline Others & 58 & 7.7 & Asteatosis/Xerosis & 365 & 100.0 \\
\hline Without skin disease & 620 & 3.5 & Others & 3,647 & 20.9 \\
\hline Without skin disease & 620 & 100.0 & Others & 3,647 & 100.0 \\
\hline
\end{tabular}




\section{Skin disorders according to gender}

The most prevalent diagnoses we found in males were: mycosis $(8.0 \%)$, non-specified eczema $(7.6 \%)$, BCC $(4.6 \%)$, atopic dermatitis $(4.1 \%)$, psoriasis $(4.0 \%)$, seborrheic keratosis (3.1\%), without skin disease (2.9\%), seborrheic dermatitis $(2.7 \%)$, acne $(2.6 \%)$, viral warts $(2.3 \%)$ and lichen simplex chronicus (2.3\%). Among females, the most common were: non-specified eczema $(7.3 \%)$, mycosis $(6.0 \%)$, without skin disease $(3.9 \%)$, seborrheic keratosis $(3.9 \%)$, BCC $(3.4 \%)$, psoriasis $(3.3 \%)$, contact dermatitis $(3.1 \%)$, atopic dermatitis $(3.0 \%)$ acne $(2.4 \%)$ and asteatosis $(2.1 \%)$ (Table 3 ).

\section{Skin disorders according to age group}

Age groups were defined according to World Health Organization criteria (0-18 years for children; 19-60 years for adults; and over 60 years for the elderly).
The most common skin disorders in children were: atopic dermatitis $(17.2 \%)$, acne $(6.2 \%)$, non-specified eczema $(5.8 \%)$, superficial mycosis $(3.7 \%)$ and viral warts $(3.6 \%)$. In adults, they were: non-specified eczema $(7.7 \%)$, superficial mycosis $(7.2 \%)$, psoriasis $(4.1 \%)$, without skin disease $(3.8 \%)$ and contact dermatitis $(3.7 \%)$. Finally, in the elderly, they were: BCC $(9.8 \%)$, seborreheic keratosis $(8.8 \%)$, non-specified eczema $(7.8 \%)$, superficial mycosis $(7.7 \%)$ and actinic keratosis $(4.7 \%)$ (Table 4$)$.

\section{Skin disorders according to destination}

The main skin disorders that resulted in follow-up at the HCFMUSP's Dermatology Division were: BCC (14.5\%), squamous cell carcinoma (4.5\%), non-specified eczema $(4.2 \%)$, superficial mycosis $(2.8 \%)$ and psoriasis $(2.5 \%)$.

TABLE 3: Main disorders according to gender

\begin{tabular}{|c|c|c|c|c|c|}
\hline Female & 10,961 & 62.8 & Male & 6,493 & 37.2 \\
\hline Non-specified eczema & 801 & 7.3 & Superficial mycosis & 519 & 8.0 \\
\hline Mycosis & 657 & 6.0 & Non-specified eczema & 493 & 7.6 \\
\hline Without skin disease & 428 & 3.9 & Basal cell carcinoma & 298 & 4.6 \\
\hline Seborrheic keratosis & 427 & 3.9 & Atopic dermatitis & 266 & 4.1 \\
\hline Basal cell carcinoma & 372 & 3.4 & Psoriasis & 259 & 4.0 \\
\hline Psoriasis & 361 & 3.3 & Seborrheic keratosis & 203 & 3.1 \\
\hline Contact dermatitis & 340 & 3.1 & Without skin disease & 189 & 2.9 \\
\hline Atopic dermatitis & 328 & 3.0 & Seborrheic dermatitis & 175 & 2.7 \\
\hline Acne & 264 & 2.4 & Acne & 168 & 2.6 \\
\hline Asteatosis/xerosis & 231 & 2.1 & Viral warts & 149 & 2.3 \\
\hline Viral warts & 221 & 2.0 & Lichen simplex chronicus & 149 & 2.3 \\
\hline Actinic keratosis & 221 & 2.0 & Contact dermatitis & 136 & 2.1 \\
\hline Melasma & 187 & 1.7 & Asteatosis/xerosis & 129 & 2.0 \\
\hline Vitiligo vulgaris & 187 & 1.7 & Vitiligo vulgaris & 112 & 1.7 \\
\hline Seborrheic dermatitis & 187 & 1.7 & Squamous cell carcinoma & 104 & 1.6 \\
\hline Residual lesions & 164 & 1.5 & Folliculitis & 92 & 1.4 \\
\hline Lichen simplex chronicus & 164 & 1.5 & Nummular eczema & 86 & 1.3 \\
\hline Melanocitic naevi & 164 & 1.5 & Pruritus & 86 & 1.3 \\
\hline Urticaria & 153 & 1.4 & Epidermal cysts & 86 & 1.3 \\
\hline Pruritus & 142 & 1.3 & Actinic keratosis & 78 & 1.2 \\
\hline Solar melanosis & 132 & 1.2 & Stasis dermatitis & 72 & 1.1 \\
\hline Epidermic cysts & 132 & 1.2 & Urticaria & 65 & 1.0 \\
\hline Nail disorders & 120 & 1.1 & Residual Lesions & 65 & 1.0 \\
\hline Acrochordon & 109 & 1.0 & Onicocriptosis & 65 & 1.0 \\
\hline Alopecia areata & 110 & 1.0 & Alopecia areata & 65 & 1.0 \\
\hline Squamous cell carcinoma & 98 & 0.9 & Drug reactions & 65 & 1.0 \\
\hline Pityriasis rosea & 98 & 0.9 & Keloid & 65 & 1.0 \\
\hline Nevi & 88 & 0.8 & Melanocitic nevi & 65 & 1.0 \\
\hline Dyshidrosis & 88 & 0.8 & Abscess, furuncle and anthrax & 50 & 0.8 \\
\hline Others & 3,989 & 36.4 & Others & 2,143 & 33.0 \\
\hline
\end{tabular}


TABLE 4: Most common skin disorders according to age group

\begin{tabular}{|c|c|c|c|c|c|c|c|c|}
\hline Child (0 - 18 years) & 2,846 & 16.3 & Adult (19 - 59 years) & 9,714 & 55.7 & Elderly (>60 years) & 4,894 & 28.0 \\
\hline Atopic dermatitis & 490 & 17.2 & Non-specified eczema & 748 & 7.7 & Basal cell carcinoma & 481 & 9.8 \\
\hline Acne & 176 & 6.2 & Superficial mycosis & 697 & 7.2 & Seborrheic keratosis & 431 & 8.8 \\
\hline Non-specified eczema & 165 & 5.8 & Psoriasis & 394 & 4.1 & Non-specified eczema & 384 & 7.8 \\
\hline Superficial mycosis & 105 & 3.7 & Without skin disease & 373 & 3.8 & Superficial mycosis & 377 & 7.7 \\
\hline Viral warts & 104 & 3.6 & Contact dermatitis & 360 & 3.7 & Actinic keratosis & 232 & 4.7 \\
\hline Vitiligo vulgaris & 91 & 3.2 & Acne & 251 & 2.6 & Without skin disease & 169 & 3.5 \\
\hline Molluscum contagiosum & 87 & 3.0 & Seborrheic dermatitis & 213 & 2.2 & Asteatosis/Xerosis & 165 & 3.4 \\
\hline Psoriasis & 82 & 2.9 & Lichen simplex chronicus & 200 & 2.0 & Squamous cell carcinoma & 161 & 3.3 \\
\hline Pityriasis alba & 81 & 2.8 & Melasma & 197 & 2.0 & Psoriasis & 143 & 2.9 \\
\hline Without skin disease & 76 & 2.7 & Viral warts & 191 & 2.0 & Lichen simplex chronicus & 101 & 2.1 \\
\hline Insect bite reactions & 66 & 2.3 & Seborrheic keratosis & 186 & 1.9 & Pruritus & 89 & 1.8 \\
\hline Seborrheic dermatitis & 61 & 2.2 & Basal cell carcinoma & 180 & 1.9 & Seborrheic dermatitis & 88 & 1.8 \\
\hline Contact dermatitis & 56 & 2,0 & Vitiligo vulgaris & 171 & 1.8 & Stasis dermatitis & 85 & 1.7 \\
\hline Alopecia areata & 53 & 1.9 & Urticaria & 169 & 1.7 & Epidermal cysts & 79 & 1.6 \\
\hline Nummular eczema & 51 & 1.8 & Asteatosis/Xerosis & 162 & 1.7 & Viral warts & 77 & 1.6 \\
\hline Melanocytic nevi & 44 & 1.6 & Residual Lesions & 158 & 1.6 & $\begin{array}{l}\text { Non-specified malignant } \\
\text { cutaneous neoplasms }\end{array}$ & 76 & 1.5 \\
\hline Residual Lesions & 43 & 1.5 & Melanocytic nevi & 148 & 1.5 & Solar melanosis & 71 & 1.4 \\
\hline Impetigo & 42 & 1.5 & Pruitus & 139 & 1.4 & Contact dermatitis & 58 & 1.2 \\
\hline Non-specified nevi & 41 & 1.4 & Folliculitis & 138 & 1.4 & Melanoma & 53 & 1.1 \\
\hline Others & 930 & 32.7 & Others & 4,639 & 47.7 & Others & 1,575 & 32.2 \\
\hline
\end{tabular}

The most common skin disorders referred to the secondary or primary health system were: psoriasis $(7.9 \%)$, superficial mycosis $(6.8 \%)$, seborrheic keratosis $(6.2 \%)$, acne $(5.9 \%)$ and non-specified eczema (5.1\%). Furthermore, the diagnoses that needed more than one appointment at the AGDD were: non-specified eczema $(16.2 \%)$, contact dermatitis $(5.6 \%)$, atopic dermatitis $(5.2 \%)$, superficial mycosis $(4.2 \%)$ and without skin disease $(4.0 \%)$ (Table 5).

\section{DISCUSSION}

Understanding the incidence of skin diseases is fundamental in making decisions regarding allocating resources for clinical care and research. Population-based studies are essential in this respect. ${ }^{8}$ At the HCFMUSP, we have already studied data on consultations and inpatient dermatology., ${ }^{4,5}$ This research is the first to analyze a triage clinic in which patients are treated at the three levels of care (primary, secondary and tertiary), seeking to complete the epidemiological analysis in our service.

The most common skin disorder in our study was eczema. In all cases, we observed non-specified eczema $(41.3 \%)$, followed by atopic dermatitis (18.8\%), contact dermatitis $(15.1 \%)$, lichen simplex chronicus $(10 \%)$, nummular eczema $(5.6 \%)$, stasis dermatitis $(4.9 \%)$ and dyshidrosis (4.4\%). Eczema is a major health problem worldwide, mainly in developed countries, where higher prevalence is influenced by socioeconomic and environmental factors. It is also associated with atopic dermatitis prevalence. Our data on eczema (18\%) are close to the proportion observed among the US population (17.1\%). ${ }^{9}$ In addition to climatic factors, the increasing use of cleaning products, especially in developing countries undergoing improvement in their populations' quality of life and socio-economic conditions, could be a reason for the comprehensive increase in eczema prevalence. ${ }^{10}$

We observed that the frequency of eczema was higher than the frequency of cutaneous infections, probably reflecting improvements in hygiene and the expansion of sewerage in Brazil. However, our data show that cutaneous infections still represent an important group of dermatoses among the population. We noted that the most common cutaneous infections were: superficial mycosis (51.5\%), viral warts $(16.2 \%)$, abscesses, furuncle and anthrax (5.2\%), molluscum contagiosum $(4.9 \%)$, herpes zoster $(3.7 \%)$, impetigo $(3.5 \%)$, herpes simplex $(3.3 \%)$, cellulitis/erysipelas $(3.1 \%)$ and leprosy $(2.7 \%)$. Indeed, leprosy is a major endemic disease in Brazil, with more than $80 \%$ of cases in the Americas. The point prevalence is 2.6 per 10,000 inhabitants, and over 40,000 new cases each year. ${ }^{11,12} \mathrm{~A}$ high prevalence of leprosy was observed in our study. We believe that this prevalence is likely not higher only because many patients were evaluated and treat- 
Table 5: Patient destination

\begin{tabular}{|c|c|c|c|c|c|}
\hline Discharged & & 32.7 & Return & & 16.0 \\
\hline Superficial mycosis & 434 & 8.5 & Non-classified eczema & 405 & 16.2 \\
\hline Non-specified eczema & 383 & 7.5 & Contact dermatitis & 141 & 5.6 \\
\hline Without skin disease & 311 & 6.1 & Atopic dermatitis & 131 & 5.2 \\
\hline Seborrheic keratosis & 298 & 5.8 & Superficial mycosis & 106 & 4.2 \\
\hline Asteatosis/Xerosis & 235 & 4.6 & Without skin disease & 100 & 4.0 \\
\hline Atopic dermatitis & 152 & 3.0 & Urticaria & 89 & 3.6 \\
\hline Seborrheic dermatitis & 149 & 2.9 & Lichen simplex chronicus & 74 & 3.0 \\
\hline Contact dermatitis & 141 & 2.8 & Pruritus & 73 & 2.9 \\
\hline Residual lesions & 134 & 2.6 & Nummular eczema & 67 & 2.7 \\
\hline Psoriasis & 117 & 2.3 & Pityriasis rosea & 62 & 2.5 \\
\hline Lichen simplex chronicus & 102 & 2.0 & Abscess, furuncle and anthrax & 56 & 2.2 \\
\hline Acne & 97 & 1.9 & Impetigo & 49 & 2.0 \\
\hline Viral warts & 88 & 1.7 & Dyshidrosis & 47 & 1.9 \\
\hline Solar melanosis & 87 & 1.7 & Herpes Zoster & 44 & 1.8 \\
\hline Pruritus & 86 & 1.7 & Seborrheic dermatitis & 43 & 1.7 \\
\hline Melasma & 82 & 1.6 & Telogen effluvium & 43 & 1.7 \\
\hline Actinic keratosis & 81 & 1.6 & Psoriasis & 40 & 1.6 \\
\hline Melanocytic nevi & 72 & 1.4 & Cellulitis/Erysipelas & 40 & 1.6 \\
\hline Folliculitis & 69 & 1.3 & Residual lesions & 39 & 1.6 \\
\hline Vitiligo vulgaris & 67 & 1.3 & Stasis dermatitis & 38 & 1.5 \\
\hline Pityriasis alba & 59 & 1.2 & Epidermal cysts & 37 & 1.5 \\
\hline Soft fibroma/acrochordon & 56 & 1.1 & Prurigo & 36 & 1.4 \\
\hline Nummular eczema & 54 & 1.1 & Insect bite reactions & 36 & 1.4 \\
\hline Insect bite reactions & 52 & 1.0 & Non-specified nail disorders & 25 & 1.0 \\
\hline Non-specified nevi & 51 & 1.0 & Non-specified drug reactions & 25 & 1.0 \\
\hline Epidermal cysts & 49 & 1.0 & Asteatosis/Xerosis & 25 & 1.0 \\
\hline Urticaria & 49 & 1.0 & Herpes simplex & 24 & 1.0 \\
\hline Non-classified nail disorders & 48 & 0.9 & Folliculitis & 23 & 0.9 \\
\hline Pityriasis rosea & 46 & 0.9 & Scabies & 18 & 0.7 \\
\hline Others & 1.469 & 28.7 & Others & 565 & 22.6 \\
\hline Follow-up at HCFMUSP & & 25.9 & Primary/secondary health system & & 25.4 \\
\hline Basal cell carcinoma & 588 & 14.5 & Psoriasis & 315 & 7.9 \\
\hline Squamous cell carcinoma & 184 & 4.5 & Superficial mycosis & 272 & 6.8 \\
\hline Not classified eczema & 168 & 4.2 & Seborrheic keratosis & 248 & 6.2 \\
\hline Superficial mycosis & 112 & 2.8 & Acne & 236 & 5.9 \\
\hline Psoriasis & 103 & 2.5 & Not classified eczema & 204 & 5.1 \\
\hline Lichen planus & 100 & 2.5 & Atopic dermatitis & 192 & 4.8 \\
\hline Non-specified malignant cutaneous neoplasms & 87 & 2.1 & Viral warts & 161 & 4.0 \\
\hline Non-specified tumor or vascular malformation & 82 & 2.0 & Vitiligo vulgaris & 157 & 3.9 \\
\hline Viral warts & 81 & 2.0 & Actinic keratosis & 135 & 3.4 \\
\hline Melanoma & 80 & 2.0 & Seborrheic dermatitis & 113 & 2.8 \\
\hline Non-specified lupus erythematosus & 68 & 1.7 & Contact dermatitis & 103 & 2.6 \\
\hline Onicocriptosis & 66 & 1.6 & Melanocytic nevi & 102 & 2.6 \\
\hline Atopic dermatitis & 66 & 1.6 & Melasma & 100 & 2.5 \\
\hline Acne & 65 & 1.6 & Lichen simplex chronicus & 91 & 2.3 \\
\hline Non-specified drug reactions & 55 & 1.4 & Soft fibroma/acrochordon & 84 & 2.1 \\
\hline Contact dermatitis & 52 & 1.3 & Epidermal cysts & 80 & 2.0 \\
\hline Keloid & 47 & 1.2 & Alopecia areata & 66 & 1.7 \\
\hline Vitiligo vulgaris & 47 & 1.2 & Stasis dermatitis & 59 & 1.5 \\
\hline Actinic keratosis & 39 & 1.0 & Asteatosis/Xerosis & 58 & 1.5 \\
\hline Molluscum contagiosum & 39 & 1.0 & Keloid & 56 & 1.4 \\
\hline Cutaneous horn & 36 & 0.9 & Without skin disease & 53 & 1.3 \\
\hline Hansen disease & 34 & 0.8 & Androgenic alopecia & 48 & 1.2 \\
\hline Non-specified collagen diseases & 34 & 0.8 & Solar melanosis & 43 & 1.1 \\
\hline Scleroderma & 34 & 0.8 & Nummular eczema & 38 & 1.0 \\
\hline Erythema nodosum & 34 & 0.8 & Folliculitis & 35 & 0.9 \\
\hline Hidradenitis & 33 & 0.8 & Pruritus & 35 & 0.9 \\
\hline Discoid lupus erythematosus & 32 & 0.8 & Callus & 34 & 0.9 \\
\hline Alopecia areata & 32 & 0.8 & Dyshidrosis & 34 & 0.9 \\
\hline Non-specified bullous diseases & & & & & \\
\hline
\end{tabular}


ed at other reference centers, and in the primary and secondary healthcare units.

Erythematous squamous conditions were also common in our study (6.8\%). Among them, psoriasis and seborrheic dermatitis were the most frequently observed, present in $54 \%$ and $30 \%$, respectively. These data were expected, since psoriasis affects approximately $2 \%$ of the population worldwide and seborrheic dermatitis is also a common skin condition, whose prevalence in adults is estimated at $5 \% \cdot{ }^{13,14,15}$ Further, the results are closer to those in Japan, where Furue et al. found a prevalence of $4.4 \%$ for psoriasis and $3.2 \%$ for seborrheic dermatitis. ${ }^{1}$ Moreover, they are in line with results obtained in Eskisehir, Turkey, where Bilgili et al. listed psoriasis as the fifth most common skin disease (5.5\%), while seborrheic dermatitis appeared in $2.2 \%$ of all cases. ${ }^{16}$ Additionally, our study was performed at a hospital with a phototherapy unit, so we expected to find a considerable prevalence of psoriasis, as many patients are referred to our clinic for this treatment. $^{1,16}$

Likewise, we observed a high incidence of malignant cutaneous neoplasm (6.1\%), 62.1\% of which were BCC cases, as expected. Data from The National Cancer Institute (INCA) show that non-melanoma skin cancer represents $25 \%$ of all cases and BCC is the most frequent type, accounting for $70 \%$ of cases. ${ }^{17}$ These findings are similar to those of Katalinic et al., who conducted a study in Germany, finding that over $80 \%$ of all skin tumors were BCC. ${ }^{18}$

Analyzing skin disorders by age group, non-specified eczema, superficial mycosis and psoriasis were frequently observed in all groups as predicted, because these disorders are very common. Atopic dermatitis was the predominant disease for children. Acne was more frequent in children and adults, as well as viral warts. BCC and squamous cell carcinoma were more common in the elderly. These findings are comparable to countries such as Japan, where the top five diseases for each age group were listed. Miscellaneous eczema occurred in every age group, whereas atopic dermatitis was among the top five diseases in individuals aged under 50. Molluscum contagiosum and impetigo were frequent among patients aged $0-10$ years. Viral warts were among the top five diseases for individuals aged 6-45 years. Acne was common in groups aged 11-35 years. Urticaria/angioedema were among the top five diseases in groups aged 11-70 years. Tinea pedis was common in groups aged above 41 years. Psoriasis appeared in the top five diseases among the middle-aged and elderly, with ages ranging from 46 to 80 years. ${ }^{1}$
The category 'without skin disease' was prevalent in all age groups. This may reflect a possible forward error and the difficulty patients face in accessing specialists in the Brazilian public health system. Specifically, when patients undergo specialized medical evaluation after a long waiting period, the disease has disappeared. This is significant and highlights the need to reduce the time taken to arrive at hospitals and adopt better referral criteria.

In our study, there was little difference in disease prevalence between men and women. The three most frequent disorders identified in males were mycosis, non-specified eczema and BCC. In females, we noted non-specified eczema, mycosis and 'without skin disease'. Contact dermatitis and acne predominated in women rather than men, similarly to the findings of Bilgili et al. and to epidemiological data published on these diseases. ${ }^{19-21}$

Regarding the destination analysis, the main skin disorders that resulted in patient follow-up at our service were: malignant cutaneous neoplasm (mainly BCC and squamous cell carcinoma), non-specified eczema, superficial mycosis, psoriasis, lichen planus, other skin tumors, viral warts, lupus, atopic dermatitis, drug reactions, keloid and vitiligo. The majority of these diseases were also the most common disorders among the population and therefore some cases were monitored at our service for residents and medical students to learn. Another reason why common diseases are monitored at the HCFMUSP is that the Brazilian health system is deficient, with a lack of specialists and infrastructure, mainly at the primary and secondary levels of care. ${ }^{7}$ Furthermore, the most complex and/ or severe cases like skin cancer and other skin tumors must be monitored at our service, since the HCFMUSP is among the biggest and best equipped tertiary, public hospitals in Latin America.

\section{CONCLUSION}

Understanding the incidence of skin diseases is fundamental in making decisions regarding resource allocation for clinical care and research. Population-based studies are essential in this respect.

In our study, the most common diseases were eczema, cutaneous infections, erythematous squamous diseases and cutaneous neoplasms. The high prevalence of some diseases with low complexity, as observed at the triage service of a tertiary hospital, partly reflects the lack of specialists and scarce resources in the primary and secondary Brazilian health system. Thus, we believe our findings can contribute to improving public health policies.] 


\section{REFERENCES}

1. Furue M, Yamazaki S, Jimbow K, Tsuchida T, Amagai M, Tanaka T, et al. Prevalence of dermatological disorders in Japan: A nationwide, cross-sectional, seasonal, multicenter, hospital-based study. J Dermatol. 2011;38:310-20.

2. Massa A, Alves R, Amado J, Matos E, Sanches M, Selores M, et al. Prevalence of cutaneous lesions in Freixo de Espada à Cinta Acta Med Port. 2000;13:247-54.

3. Peñate $Y$, Guillermo N, Melwani P, Martel R, Borrego L. Dermatologists in Hospital Wards: An 8-year Study of Dermatology Consultation. Dermatology. 2009;219:225-31.

4. Mancusi S, Festa Neto C. Inpatient dermatological consultations in a university hospital. Clinics (Sao Paulo). 2010;65:851-5.

5. de Paula Samorano-Lima L, Quitério LM, Sanches JA Jr, Neto CF. Profile of patients and characteristics of admissions to a tertiary dermatology ward (unit) in São Paulo, Brazil. Int J Dermatol. 2014;53:685-91.

6. Bale J, Chee P. Inpatient dermatology: Pattern $f$ admissions and patients' characteristics in an Australian hospital. Australas J Dermatol. 2014;55:191-5.

7. Paim J, Travassos C, Almeida C, Bahia L, Macinko J. The Brazilian health system: history, advances, and challenges. Lancet. 2011;377:1778-97.

8. Andersen LK, Davis MD. The epidemiology of skin and skin-related diseases: a review of population-based studies performed by using the Rochester Epidemiology Project. Mayo Clin Proc. 2013;88:1462-7.

9. Hanifin JM, Reed ML; Eczema Prevalence and Impact Working Group. A population-based survey of eczema prevalence in the United States. Dermatitis. 2007;18:82-91.

10. Williams H, Stewarts A, von Mutius E, Cookson W, Anderson HR; International Study of Asthma and Allergies in Childhood (ISAAC) Phase One and ThreeStudy Groups. Is eczema really on the increase worldwide? J Allergy Clin Immunol. 2008;121:947-54.e15

11. Martelli CM, Stefani MMA, Penna GO, Andrade AL. Endemias e epidemias brasileiras, desafios e perspectivas de investigação científica: hanseníase. Rev Bras Epidemiol. 2002;5:273-85.

12. Tabnet.datasus.gov.br [Internet]. Ministério da Saúde. Datasus. Sistema de Informação de Agravos de Notificação. Departamento de Vigilância Epidemiológica. Avaliação da Prevalência da Hanseníase no Brasil [acesso 28 abr 2014]. Disponivel em: http://tabnet.datasus.gov.br/cgi/deftohtm.exe?idb2012/ d09b.def

13. $\mathrm{Ni} \mathrm{C}$, Chiu MW. Psoriasis and comorbidities: links and risks. Clin Cosmet Investig Dermatol. 2014;7:119-32.

14. Dessinioti C, Katsambas A. Seborrheic dermatitis: etiology, risk factors, and treatments: facts and controversies. Clin Dermatol. 2013;31:343-51.

15. Bukvić Mokos Z, Kralj M, Basta-Juzbašić A, Lakoš Jukić I. Seborrheic dermatitis: an update. Acta Dermatovenerol Croat. 2012;20:98-104.

16. Bilgili ME, Yildiz H, Sarici G. Prevalence of skin diseases in a dermatology outpatient clinic in Turkey. A cross-sectional, retrospective study J Dermatol Case Rep. 2013;7:108-12.

17. Inca.gov.br [Internet]. Instituto Nacional de Cancer José Alencar Gomes da Silva. Tipos de câncer de pele não melanoma. Rio de Janeiro: Inca; 2012. [acesso 28 abr 2014]. Disponivel em: http://www2.inca.gov.br/wps/wcm/connect/ tiposdecancer/site/home/pele_nao_melanoma

18. Katalinic A, Kunze U, Schäfer T. Epidemiology of cutaneous melanoma and non-melanoma skin cancer in Schleswig-Holstein, Germany: incidence, clinical subtypes, tumour stages and localization (epidemiology of skin cancer). $\mathrm{Br} \mathrm{J}$ Dermatol. 2003;149:1200-6.

19. Silverberg JI, Silverberg NB. Epidemiology and extra-cutaneous comorbidities of severe acne in adolescence: A US population-based study. $\mathrm{Br} \mathrm{J}$ Dermatol. 2014; $170: 1136-42$.

20. Thyssen JP, Linneberg A, Menné T, Johansen JD. The epidemiology of contact allergy in the general population-prevalence and main findings. Contact Dermatitis. 2007; $57: 287-99$

21. Peiser M, Tralau T, Heidler J, Api AM, Arts JH, Basketter DA, et al. Allergic contact dermatitis: epidemiology, molecular mechanisms, in vitro methods and regulatory aspects. Current knowledge assembled at an international workshop at BfR, Germany. Cell Mol Life Sci. 2012;69:763-81.
MAILING ADDRESS:

Alyne Korukian Freiberg

Secretaria do Departamento de Dermatologia da FMUSP

Sala $3068-3^{\circ}$ andar

Instituto Central da Hospital das Clínicas da FMUSP

Av. Dr. Enéas de Carvalho Aguiar, 255

Cerqueira César

05403-000 - São Paulo - SP

Brazil

E-mail: alyne.freiberg@gmail.com

How to cite this article: Bertanha F, Nelumba E, Freiberg AK, Samorano LP, Festa Neto C. Profile of patients admitted to a triage dermatology clinic at a tertiary hospital in São Paulo, Brazil. An Bras Dermatol. 2016;91(3):318-25. 